\title{
Modelo estructural de las competencias de innovación en Latinoamérica. Evidencia en México, Colombia y Bolivia.
}

\section{Structural model of innovation competencies in Latin America. Evidence in Mexico, Colombia and Bolivia}

\author{
Paloma Daniela Gómez Álvarez ${ }^{1}$, Marco Alberto Núñez Ramírez ${ }^{2}$, \\ Irma Guadalupe Esparza García ${ }^{3}$
}

${ }^{1}$ Privada 1, No. 7512, Valle del Sol, cel. 6442089748 (correo: dnieelaga@ hotmail.com) Estudiante de la Maestría en Gestión Organizacional. Instituto Tecnológico de Sonora, https://orcid.org/0000-0002-4064-1369

${ }^{2}$ Marco Alberto Núñez Ramírez, Instituto Tecnológico de Sonora, Departamento de Ciencias Administrativas, 5 de febrero 818 Sur, Ciudad Obregón, Sonora, México (correo marco.nunez@itson.edu.mx). Tel. 6444100900 Ext. 2744. http://orcid.org/0000-0001-5825-4482

\section{${ }^{3}$ Irma Guadalupe Esparza García}

Instituto Tecnológico de Sonora, Departamento de Ciencias Administrativas, 5 de febrero 818 Sur, Ciudad Obregón, Sonora, México (correo: iesparza@itson.edu.mx). Tel. 6444100900 Ext. 2734 https://orcid.org/0000-0002-3715-0761

\section{DOI https://doi.org/10.46589/rdiasf.vi36.426}

Recibido 4 de junio 2021.

Aceptado 30 de septiembre 2021

Publicado 31 de octubre 2021

\section{Resumen}

El objetivo de la presente investigación es determinar cómo influyen las competencias de innovación individual e interpersonal sobre la creación de redes dentro del contexto latinoamericano. Se llevó a cabo una investigación cuantitativa y correlacional y, además, se elaboró un modelo estructural a través del programa SmartPLS. Aquí, mediante una muestra conformada por 153 participantes de México $(n=40)$, Bolivia $(n=85)$ y Colombia $(n=28)$, 
se encontró que sólo la competencia de innovación interpersonal tiene un impacto positivo y significativo sobre la creación de redes. En conclusión, la aportación del presente documento muestra que el desarrollo de la innovación en una región colectivista como latinoamericana, requiere de una interpretación cultural.

Palabras clave: Competencias para la innovación, Latinoamérica, Modelación con Ecuaciones Estructurales.

\begin{abstract}
The objective of this research is to determine how individual and interpersonal innovation competencies influence networking within the Latin American context. A quantitative and correlational investigation was carried out and, in addition, a structural model was elaborated through the SmartPLS program. Here, through a sample made up of 153 participants from Mexico $(n=40)$, Bolivia $(n=85)$ and Colombia $(n=28)$, it was found that only interpersonal innovation competence has a positive and significant impact on the creation of networks. In conclusion, the contribution of this document shows that the development of innovation in a collectivist region such as Latin America requires a cultural interpretation.
\end{abstract}

Keywords: Competences for innovation, Latin America, Modeling with Structural Equations.

\title{
Introducción
}

La innovación se ha vuelto un campo muy amplio de investigación dentro de las ciencias administrativas. Si bien Schumpeter (1994), como pionero de este campo, estudió la innovación en relación a una actitud creativa que contrastaba con el comportamiento rutinario de las personas; además, expresaba que la innovación tenía diversos significados, entre los cuales se encontraba lo siguiente: a) la introducción de un nuevo producto de calidad; b) la generación de un método de producción; c) la apertura de nuevos mercados; d) el apoderarse de una nueva fuente de cadena de suministro; y, finalmente, e) la realización de una nueva organización. 
Es importante resaltar que dicha variable también se ha asociado con los trabajos de Taylor (1975), en los cuales este autor introdujo cambios con la llegada de su teoría científica de la administración, al implementar nuevas prácticas y métodos de trabajo orientados hacia la eficiencia organizacional. Otro referente histórico de la innovación dentro de la teoría administrativa se encuentra en los trabajos de Fayol (1975), donde se le ubica en la llegada de nuevas mecánicas de trabajo para facilitar las funciones administrativas y el gobierno de las empresas. Además, otro teórico que se asocia es Weber (1992), con su contribución de novedosas prácticas administrativas al implementar una estructura burocrática, la cual cambiaría la administración para enfocarse en tareas que se regularan por reglas y procedimientos.

Por otro lado, a pesar de que existen diversos tipos de innovación, según Schumpeter (1994), Rochina y Rodríguez (2019), los categorizan en cuatro: proceso, producto, organizativas o administrativas y de marketing. En el caso de la perspectiva organizacional, se relaciona con la introducción de nuevos y novedosas prácticas de negocio, nuevos métodos para organizar el trabajo, el crecimiento de las empresas y el tamaño (Corsi et al. 2019), dentro de este tipo de innovación, se destacan las habilidades y competencias que se tienen para adquirir o transferir conocimientos.

Desde el enfoque de recursos y capacidades, las competencias se han catalogado como estrategias para alcanzar un crecimiento y competitividad, viéndose en las capacidades como fuente principal para el desarrollo de habilidades, procesos y conocimiento (Barney et al. 2001), los cuales llegan a ser fundamentos de innovación. Por otra parte, estas capacidades se han desarrollado en base a los recursos que se tienen (Fierro y Mercado, 2012), siendo ambos puntos de partida para establecer relaciones interorganizacionales e implementar redes organizacionales, donde se encuentra una gama de flujo de información, de productos, servicios y personal (Evan, 1967).

Rangel (2015) expresa que las competencias se han convertido en un tema controvertido y de gran interés para las universidades y la educación en la actualidad, de manera similar, Muñoz et al. (2016) argumentan que las competencias son todas aquellas actitudes, conocimientos y habilidades que permiten el diseño de funciones exitosas que, además, llegan a ser transferibles. Sin embargo, las competencias interpersonales han sido 
situadas por debajo, es decir, mostraban debilidad en cuanto a la formación social de los docentes.

Olivares et al. (2019), también, concuerdan con que las competencias se han vuelto un desafío para las universidades, ya que estas se miran presionadas a inculcar competencias trasversales a los estudiantes, las cuales permitan poder adaptarse a los entornos cambiantes de manera profesional y personal. Por otra parte, Ochoa et al. (2011) menciona que las capacidades contribuyen a responder de manera adecuada a los desafíos del entorno, así como aportar con habilidades que fomenten la innovación de las organizaciones, dando como resultados tendencias internacionalizadas durante las operaciones en el mercado.

En el caso de este tipo de competencias, Madrigal et al. (2019) estudiaron la importancia de la capacidad emprendedora e innovadora de 522 estudiantes universitarios de México, con la finalidad de aportar insumos para formación de programas que permitieran fomentar la innovación. Es así que, en dicho estudio, se encontró que las universidades tenían un impacto positivo en el desarrollo de habilidades individuales, interpersonales y la creación de redes, debido a que, en dichos centros educativos, se fomenta la promoción integral de estrategias que contribuyen al emprendimiento. Aquí, además, se encontró que las habilidades individuales son el punto central para el desarrollo de habilidades interpersonales, y, por lo tanto, la creación de redes colaborativas. Por último, se halló que los jóvenes que participaban en programan extracurriculares, desempeñaban mayores habilidades de innovación.

Por otro lado, Madrigal y Nuñez (2021) retoman dicha metodología, la cual fue desarrollada en el proyecto INCODE del Programa de Aprendizaje Permanente en la Unión Europea por Watts et al. (2013), y consta de 25 ítems que integran tres dimensiones: 1) competencias individuales (doce ítems); 2) competencias interpersonales (ocho ítems), 3) y competencias para la creación de redes (cinco ítems). Este aspecto se detalla con mayor profundidad dentro del método.

\section{Planteamiento del problema}

Si bien, Latinoamérica sobresale a nivel mundial por ser la región con el nivel más alto de la TEA (Tasa de actividad emprendedora), según estadísticas publicadas por el Monitor Global 
de Emprendimiento ([GEM], 2018), todavía existe muchas áreas de oportunidad en cuanto al desarrollo de la innovación (Banco Interamericano para el Desarrollo [BID], 2010). En este contexto, el fomento de la innovación puede ser explicada desde un enfoque individual. Pues, como dice Gartner (1988), los emprendedores no pueden ser definidos a través de sus personalidades, sino a través de lo que hacen: generar organizaciones.

Por otro lado, Drucker (1985), al reflexionar acerca de la principal característica del emprendedor, concluye que lo que define al emprendimiento exitoso es la innovación basada en conocimiento. En esta misma sintonía, décadas atrás Schumpeter (1943), conceptualizó al emprendedor o (intrapreneur), como un personaje que genera un "rompimiento" del mercado a través el ejercicio de la creatividad y la innovación. Hoy en día, dichos postulados siguen vigentes. No obstante, una posible reinterpretación de tales conceptos puede ser dada a través de un enfoque cultural.

Hofstede y Hofstede (2005) caracterizan a las culturas latinoamericanas como colectivistas y jerárquicas. Esto muestra dos aristas: 1) por un lado, las culturas organizacionales en esta región se destacan por el compañerismo y comunicación, a diferencia de los valores de países de primer mundo (en especial anglosajones-protestantes), lo cuales se distinguen por ser individualistas; 2) la estructuras jerárquicas o burocráticas, aunque tienden a buscar la eficiencia en todo momento, no promueven el fomento de la innovación y la creatividad (Cameron y Quinn, 2006).

Retomando el punto número uno, en los trabajos de Weber (1992), es posible encontrar una fuerte relación entre individualismo -característica del capitalismo- y desarrollo económico; mientras que este mismo autor pone énfasis en la burocracia como estructura organizacional basada en normas y jerarquía (Dávila, 2001); donde se destaca que este tipo organizaciones funciona actualmente. No obstante, en un mundo cambiante como el actual, se requiere el fomento de culturas organizaciones flexibles, donde se premie la creatividad y la innovación.

Sin embargo, a pesar de lo anterior, muchas de las organizaciones de Latinoamérica son jerárquicas y rígidas. ¿Por qué sucede esto? Una posible interpretación puede darse a través de la teoría interorganizacional, la cual se centra en rol que cada miembro de una organización debe desempeñarse dentro de un status específico en función de su relación con 
otras organizaciones (Evan, 1967), sin intervenir con algún cambio en estos roles. Derivado de esta rígida practica de rol, Provan y Milward (1995) diseñan una teoría preliminar sobre la eficacia de la red interorganizacional, cuyo objetivo radica en que las organizaciones, para lograr generar un objetivo en común, deben de establecer relaciones con otras organizaciones de manera formal.

La implementación de redes interorganizacionales conlleva la dinámica de que todas las organizaciones implicadas logren sus objetivos, fomentando una relación de cooperación, estructuración y transferencia de conocimientos (Provan y Milward, 1995). Esto, entendiéndose que la red interorganizacional es una serie de combinación de empresas y organismos, que tienen por objetivo incrementar su respuesta de demanda de servicio, también se mira implicada el incremento de innovación, ya que fusionan y transfieren entre sí, sus conocimientos, métodos, procedimientos, entre otros.

En estudios previos, Madrigal et al. (2019), al estudiar a estudiantes universitarios de México, encontraron que cada una de estas competencias (i.e., individual, interpersonal y creación de redes), son independientes entre sí. Dichos autores, además, citan que éstas tienen una fuerte relación entre sí; es decir, que, siguiendo con la propuesta de Watts et al. (2013), teniendo como propósito el generar competencias innovadoras -intangibles-, este modelo debe ser visto de manera holista, en especial, desde lo teórico.

Watts et al. (2013) proponen que la innovación -vista como fenómeno- es el resultado de tres aspectos: 1) la parte individual, donde la persona se distingue, ya sea por aspectos propios de su personalidad, como lo comentaba McClelland (1961), o por su actuar (Gartner, 1988); 2) la interpersonal, donde la persona, a través de su interacción social desarrolla su creatividad, como es el caso del surgimiento de grandes empresas como Apple o Facebook, donde hay un común denominador: varias personas talentosas unieron sus capacidades; y, finalmente, 3) la creación de redes que, en esencia, se basa en el desarrollo de un capital relacional.

De hecho, diferentes teóricos del capital intelectual, como Bueno (2013), ponen énfasis en este activo intangible, el cual genera valor a través de la relación con los clientes, proveedores, gobierno, universidades, competidores, entre otros. Dentro del contexto latinoamericano, las redes y la innovación son dos temas que requieren atención (GEM, 
2018); por lo tanto, ¿cómo podría mejorar este aspecto a partir de la parte individual e interpersonal? A partir de todo esto surge la presente interrogante: ¿Cómo influyen las competencias de innovación individual e interpersonal sobre la creación de redes dentro del contexto latinoamericano? Con el fin de dar una respuesta a dicha cuestión, a continuación, se plantean las siguientes hipótesis:

$H_{1}$ La competencia de innovación individual influye de manera significativa y positiva sobre la creación de redes dentro del contexto latinoamericano.

$\mathrm{H}_{2}$. La competencia de innovación interpersonal influye de manera significativa y positiva sobre la creación de redes dentro del contexto latinoamericano

Finalmente, se muestra como objetivo lo siguiente: determinar cómo influyen las competencias de innovación individual e interpersonal sobre la creación de redes dentro del contexto latinoamericano, a través de un modelo estructural en SmartPLS.

\section{Método}

La presente investigación fue de tipo cuantitativa, con un alcance correlacional. Además, su diseño fue no experimental, con un corte transversal. En el sentido de cuantitativo se entiende que este tipo de investigación se usa con el apoyo de la recolección de datos, con el propósito de probar una hipótesis, es decir, se utiliza la medición numérica y análisis estadísticos, en cuanto a un alcance correlacional, se refiere a aquellos estudios que tienen la finalidad de responder a preguntas de investigación, además de asociar e identificar si existe una relación entre variables. Al hablar de no experimental, se expresa que son estudios donde no se manipulan variables, únicamente se observan en su medio ambiente natural y posteriormente se analizan; y, por último, se habla de corte transversal ya que se recolecto la muestra en un solo momento (Hernández-Sampieri et al., 2014).

\section{Participantes.}

Se obtuvo una muestra no probabilística por conveniencia conformada por 153 participantes de México $(n=40)$, Bolivia $(n=85)$ y Colombia $(n=25)$. Además, de estos el $55 \%$ era del género masculino, mientras que el $45 \%$ femenino. 


\section{Instrumento}

Con el fin de medir la variable de estudio, se empleó un cuestionario construido por Watts et al. (2013), el cual es denominado como INCODE Barometer and User Guide, en la adaptación propuesta por Madrigal y Núñez (2020), donde se mide tres tipos de competencia de innovación: competencias individuales, competencias interpersonales y creación de redes. La operacionalización de dicha variable puede ser observada en la Tabla 1. Cabe señalar que la escala empleada fue Likert de 10 puntos, cuyos valores oscilaban entre 1 (no observado) a 10 (excelente).

Tabla 1. Operacionalización de la variable

\begin{tabular}{|c|c|c|}
\hline Variable & Dimensiones & Ítems \\
\hline $\begin{array}{l}\text { Competencias } \\
\text { para la } \\
\text { innovación }\end{array}$ & $\begin{array}{l}\text { Competencias } \\
\text { individuales }\end{array}$ & $\begin{array}{l}\text { 1. Propone ideas que son adecuadas para la } \\
\text { tarea } \\
\text { 2. Propone ideas creativas } \\
\text { 3. Propone nuevas formas para } \\
\text { implementar las ideas } \\
\text { 4. Evalúa ventajas y desventajas de sus } \\
\text { acciones } \\
\text { 5. Identifica relaciones entre diferentes } \\
\text { componentes de la tarea } \\
\text { 6. Enfoca la tarea desde diferentes puntos } \\
\text { de vista } \\
\text { 7. Utiliza los recursos disponibles } \\
\text { ingeniosamente } \\
\text { 8. Prevé cómo se desarrollarán los eventos } \\
\text { 9. Muestra entusiasmo } \\
\text { 10. Persigue sus metas de forma persistente } \\
\text { 11. Toma riesgos razonables } \\
\text { 12. Orienta la tarea hacia los objetivos }\end{array}$ \\
\hline
\end{tabular}




\begin{tabular}{|c|c|}
\hline $\begin{array}{l}\text { Competencias } \\
\text { interpersonales }\end{array}$ & $\begin{array}{l}\text { 13. Transmite sus ideas de forma efectiva } \\
\text { 14. Escucha a sus compañeros de equipo } \\
\text { 15. Establece relaciones constructivas de } \\
\text { grupo a través del diálogo } \\
\text { 16. Colabora activamente } \\
\text { 17. Contribuye al funcionamiento del grupo } \\
\text { 18. Toma la iniciativa } \\
\text { 19. Impulsa a otros a actuar } \\
\text { 20. Enfrenta conflictos con flexibilidad para } \\
\text { alcanzar acuerdos }\end{array}$ \\
\hline Creación de redes & $\begin{array}{l}\text { 21. Aplica valores éticos } \\
\text { 22. Toma en cuenta las implicaciones de la } \\
\text { tarea para la sociedad } \\
\text { 23. Es capaz de trabajar en ambientes } \\
\text { multidisciplinarios } \\
\text { 24. Es capaz de trabajar en ambientes } \\
\text { multiculturales } \\
\text { 25. Utiliza sus redes de contactos para lograr } \\
\text { metas }\end{array}$ \\
\hline
\end{tabular}

Fuente. Madrigal y Núñez (2021, pp. 18-19), con base en Watts et al. (2013).

\section{Confiabilidad y Validez.}

Considerando que la confiabilidad, según Ho (2008), es la consistencia interna de un constructo o variable, en la Tabla 2, se puede observar que, luego de haber realizado las pruebas alpha de Cronbach y Fiabilidad compuesta, se obtuvieron resultados favorables. Es decir, en las tres dimensiones se encontraron valores superiores a 0.70 , tanto para la $(\alpha)$ como para la fiabilidad compuesta, que, según Martínez y Fierro (2018), es el valor mínimo 
aceptable en ambas pruebas estadísticas. Resultados similares son reportados por Madrigal et al. (2019).

En cuanto a la validez, la cual se refiere a la cualidad donde el instrumento mide lo que debe de medir (Cresswell, 2014), dentro de la presente investigación se llevaron a cabo tres pruebas para medir dicha cualidad psicométrica. Primeramente, por medio de la realización de la modelación de ecuaciones estructurales en SmartPLS, se obtuvo la Varianza Extraída Media (AVE) para medir la validez convergente del cuestionario. Con el fin de cumplir con dicha prueba, de acuerdo con Hair et al. (2014), se requiere obtener un valor superior a .50, y, como se observa en la Tabla 2, en las tres dimensiones se cumple esto. En segundo lugar, se analizó la validez discriminante -la cual se refiere a la diferenciación entre las dimensiones (APA, 2010)-, cuyos indicadores, según Martínez y Fierro (2018), se refieren a cuando AVE es mayor a la correlación entre las dimensiones, lo cual es conocido como el criterio Fornell-Larcker (Tabla 3).

Tabla 2. Confiabilidad y validez convergente

\begin{tabular}{|l|r|r|r|r|}
\hline & $\begin{array}{c}\text { Alfa de } \\
\text { Cronbach }\end{array}$ & rho_A & $\begin{array}{r}\text { Fiabilidad } \\
\text { compuesta }\end{array}$ & $\begin{array}{c}\text { Varianza Extraída } \\
\text { media (AVE) }\end{array}$ \\
\hline Creación de redes & .853 & .855 & .888 & .532 \\
\hline Individual & .928 & .933 & .939 & .506 \\
\hline Interpersonal & .891 & .896 & .914 & .571 \\
\hline
\end{tabular}

Nota. Elaboración propia.

Tabla 3. Validez discriminante (criterio Fornell-Larcker)

\begin{tabular}{|l|r|r|r|}
\hline & 1 & 2 & 3 \\
\hline 1. Creación de redes & $(.729)$ & & \\
\hline 2. Individual & .555 & $(.778)$ & \\
\hline 3. Interpersonal & .710 & .747 & $(.756)$ \\
\hline
\end{tabular}

Nota. Elaboración propia.

Finalmente, como se puede apreciar en la Tabla 4, luego de realizar la prueba Heterotrait-Monotrait Ratio (HTMT), se obtuvieron favorables; la cual es empleada, 
también, para evaluar la validez discriminante. En conclusión, respecto a la validez, el cuestionario cumplió adecuadamente. Dichos hallazgos coinciden con lo reportado por Madrigal et al. (2019), quienes encontraron que dichas competencias se diferencian significativamente entre sí; aspecto que converge con el concepto de "validez discriminante".

Tabla 4. Validez discriminante (HTMT)

\begin{tabular}{|l|r|r|r|}
\hline & 1 & 2 & 3 \\
\hline 1. Creación de redes & & & \\
\hline 2. Individual & .606 & & \\
\hline 3. Interpersonal & .808 & .822 & \\
\hline
\end{tabular}

Nota. Elaboración propia.

Luego de aplicar una prueba piloto, el cuestionario fue adaptado para ser aplicado con participantes de tres países. Posteriormente, se solicitó autorización para aplicar el instrumento a autoridades de las universidades participantes: Instituto Tecnológico de Sonora (México), Universidad ICESI (Colombia) y UAGRM Business School (Bolivia). Después de explicar el objetivo y carácter de confidencialidad de la investigación, el instrumento fue autoadministrado en dichas instituciones educativas.

Para el análisis de los datos, se empleó SPSS. Posteriormente, con el fin de poner a prueba las hipótesis planteadas, se utilizó el programa SmartPLS. A continuación, se muestran los resultados obtenidos, tanto los descriptivos como los inferenciales.

\section{Resultados}

Esta sección es presentada en dos partes. En la primera, se muestran los resultados descriptivos. En la tabla 4, es posible observar dichos indicadores para cada uno de los ítems del cuestionario. Es importante señalar los valores VIF mostraron resultados menores a 4.0, lo cual refleja que no existen problemas de multicolinealidad (Hair, et al., 2014). Este último indicador es valioso para mostrar la pertinencia del Modelo estructural que se llevó cabo para poner a prueba las hipótesis de investigación. 
Tabla 4. Resultados descriptivos

\begin{tabular}{|c|c|c|c|c|c|c|}
\hline Indicador & $M$ & $M e$ & Min & $\operatorname{Max}$ & $D E$ & $V I F$ \\
\hline IDEAS & 6.92 & 7.0 & 1.0 & 10.0 & 2.27 & 3.261 \\
\hline CREATIVIDAD & 6.83 & 7.0 & 1.0 & 10.0 & 2.25 & 3.808 \\
\hline NUEVAS SFORMAS & 6.83 & 7.0 & 1.0 & 10.0 & 2.01 & 3.720 \\
\hline EVALUA VENTAJAS & 6.69 & 7.0 & 1.0 & 10.0 & 2.13 & 2.563 \\
\hline RELACIONES & 6.49 & 7.0 & 1.0 & 10.0 & 1.80 & 2.542 \\
\hline ENFOCA TAREA & 6.69 & 7.0 & 1.0 & 10.0 & 1.88 & 2.124 \\
\hline RECURSOS & 6.98 & 7.0 & 2.0 & 10.0 & 1.99 & 2.391 \\
\hline EVENTOS & 7.06 & 8.0 & 1.0 & 10.0 & 2.05 & 1.713 \\
\hline ENTUSIASMO & 7.55 & 8.0 & 1.0 & 10.0 & 2.09 & 2.664 \\
\hline METAS & 7.74 & 8.0 & 1.0 & 10.0 & 2.10 & 2.444 \\
\hline RIESGOS & 7.11 & 8.0 & 1.0 & 10.0 & 2.09 & 1.497 \\
\hline ORIENTACION TAREA & 7.25 & 8.0 & 1.0 & 10.0 & 1.99 & 1.956 \\
\hline IDEAS & 6.70 & 7.0 & 1.0 & 10.0 & 2.11 & 2.218 \\
\hline ESCUCHA & 7.46 & 8.0 & 3.0 & 10.0 & 1.80 & 2.426 \\
\hline RELACIONES & 7.44 & 8.0 & 2.0 & 10.0 & 1.80 & 2.702 \\
\hline COLABORACION & 7.72 & 8.0 & 3.0 & 10.0 & 1.70 & 2.823 \\
\hline CONTRIBUCION & 7.70 & 8.0 & 1.0 & 10.0 & 1.74 & 2.411 \\
\hline INICIATIVA & 7.74 & 8.0 & 1.0 & 10.0 & 1.93 & 1.844 \\
\hline ACTUAR & 7.84 & 8.0 & 2.0 & 10.0 & 1.82 & 1.744 \\
\hline CONFLICTOS & 7.39 & 8.0 & 1.0 & 10.0 & 1.78 & 1.872 \\
\hline VALORES ETICOS & 7.98 & 8.0 & 1.0 & 10.0 & 1.88 & 1.563 \\
\hline TAREAS & 7.48 & 8.0 & 2. & 10.0 & 1.79 & 1.979 \\
\hline AMBIENTES MULTIDISCIPLINARIO & 7.90 & 8.0 & 2.0 & 10.0 & 1.80 & 2.025 \\
\hline AMBIENTES MULTICULTURALES & 7.96 & 8.0 & 1.0 & 10.0 & 1.81 & 2.230 \\
\hline UTILIZA REDES & 7.66 & 8.0 & 1.0 & 10.0 & 2.19 & 1.617 \\
\hline
\end{tabular}

Fuente. $M=$ Media; $M e=$ Mediana; $M i n=$ Mínimo; $M a x=$ Máximo; $V I F=$ Factor de Inflación de la Varianza.

En cuanto a los resultados inferenciales, se encontró dentro el modelo estructural que la competencia interpersonal influye sobre la creación de redes de manera significativa y positiva $\left(\beta=.668 ; t=7.691 ; p<0.001 ; \mathrm{Q}^{2}=.254\right)$. No obstante, la relación entre la 
competencia individual y la creación de redes no fue significativa estadísticamente hablando, según los resultados obtenidos mediante el programa SmartPLS 3 (Ringle et al., 2015).

\section{Figura 1. Modelo Estructural}

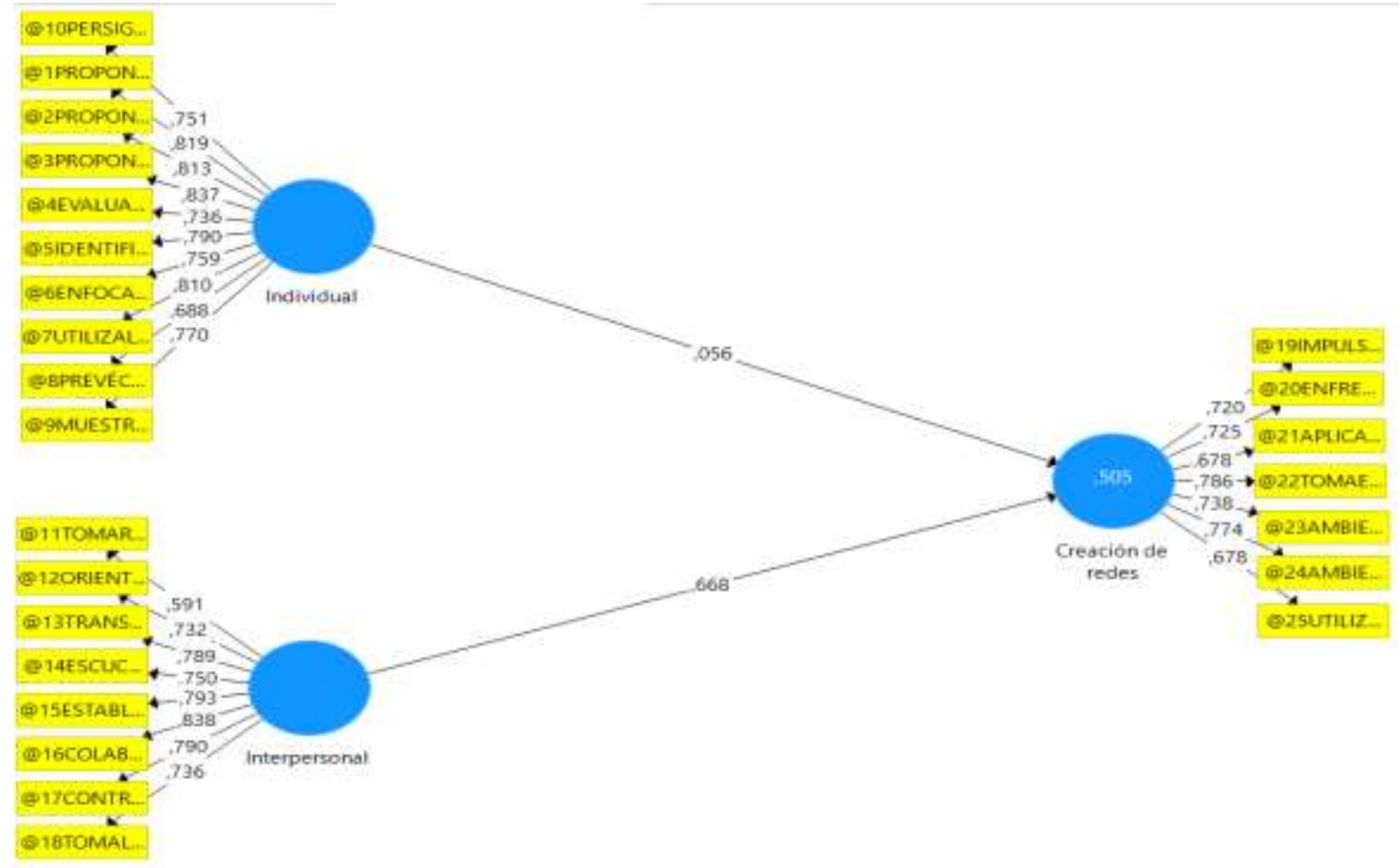

\section{Discusión}

Según Aznar et al. (2015), el INCODE es una propuesta metodológica que surgió como herramienta para desarrollar la innovación entre los estudiantes universitarios; no obstante, se requiere que dichos aspectos sean estudiados de manera integral. Asimismo, los hallazgos mostrados son una continuación a otros estudios previos, donde el INCODE ha sido empleado como una herramienta valiosa.

Primeramente, esto concuerda con lo presentado por Madrigal et al. (2019), quienes, en un estudio realizado en diferentes universidades de México (e.g., Universidad de Guadalajara, Universidad Veracruzana, Sistema Tecnológico Nacional, entre otras), encontraron que dichas dimensiones tienen una fuerte relación entre sí. De este modo, los presentes resultados no solo confirman esto, sino que muestran que la creación de redes capital relacional-, es un área de oportunidad dentro de la educación superior orientada hacia la formación de emprendimiento e innovación. 


\section{Conclusiones}

Dentro de la presente investigación, se pudo soportar empíricamente solo una de las dos hipótesis planteadas. Los hallazgos mostraron que la parte interpersonal juega un rol relevante para fomentar redes, las cuales a su vez - de manera conjunta-, pueden ser herramientas para el desarrollo de la innovación, ya sea de manera dentro de una organización (intrapreneur) o fuera de ésta (entrepreneur).

La innovación es una respuesta a los cambios propios de la Cuarta revolución industrial (Madrigal y Nuñez, 2020). No obstante, Latinoamérica es una región donde todavía se requiere trabajar más en el desarrollo de competencias para innovar dentro del emprendimiento y en la práctica administrativa (GEM, 2018). Aunque esto ya había sido promovido por Drucker (1985), todavía queda mucho por estudiar, en especial, en una región latinoamericana, donde la innovación podría venir a aportar desarrollo.

A través de la muestra conformada por participantes de México, Bolivia y Colombia, fue posible observar que se requiere mejorar el desarrollo de la parte individual e interpersonal para impactar en la creación de redes. En culturas colectivistas y rígidas como las latinoamericanas (Hostede y Hostede, 2005), la promoción de la competencia individual para innovar podría aportar para la creación de redes. Es así que los hallazgos podrían ser vistos a la luz de las teorías interorganizacionales (Provan y Milward, 1995) y, además, de recursos y capacidades (Barney, 1991), las cuales -en conjunto- promueven el desarrollo de activos intangibles, donde la parte social juega un rol trascendental. Es así que, para fortalecer la creación de redes dentro de la innovación, se requiere, también, poner énfasis en lo individual.

Finalmente, dentro de las limitaciones del estudio, se resalta el tipo de muestreo -no probabilístico- y el tamaño de la muestra, aspectos que pueden mejorarse para próximas investigaciones. Se destaca que ambos aspectos no fueron impedimento para validar el cuestionario ni para poner a prueba las hipótesis mediante el modelo estructural que, a grandes rasgos, mostró resultados favorables en cuanto a los niveles de explicación y ausencia de multicolinealidad. También, aunque esto no afectó a la validez, sería recomendable revisar la pertinencia de los ciertos indicadores que obtuvieron cargas externas 
-dentro del modelo estructural- cuyos valores fueron menores a .70. Esto podría verificarse con una muestra mayor.

\section{Referencias}

Asociación Americana de Psicología ([APA], 2010). APA. Diccionario Conciso de Psicología. Manual Moderno.

Aznar M., L. E., Montero F, B., Pérez P., M. J., Watts, F., García C., A., Marin G., J. A. (2015). De proyecto INCODE a FINCODA: Utilización del Barómetro INCODE en Alumnos Universitarios y Empleados con Competencias de Innovación: En In-Red Congreso de Innovación Educativa y Docencia.

Banco Interamericano para el Desarrollo ([BID], 2010). Science, Technology, and Innovation in Latin America and the Caribbean. A Statistical Compendium of Indicators. Inter-American Development Bank.

Barney, J. B. (1991). Firm Resources and Sustained Competitive Advantage. Journal of Management, 17, 99-120.

Barney, J., Wright, M., \& Ketchen, D. (2001). The Resource-Based View of the Firm: Ten Years After 1991. Journal of Management, 27, 625-641. https://doi.org/10.1177/014920630102700601

Bueno-CIC. (2003). Modelo Intellectus: Medición y Gestión del Capital Intelectual. DICREA Creaciones Gráficas.

Cameron, K.S. y Quinn, R.E. (2006). Diagnosing and Changing Organizational Culture (4ta ed.). JosseyBass.

Corsi, C., Prencipe, A., \& Capriotti, A. (2019). Linking Organizational Innovation, Firm Glowth and Firm Size. Management Research, 17(1), 24-49. https://doi.org/10.1108/CMS-09-2018-0676

Creswell, J. W. (2014). Research design: Qualitative, quantitative and mixed methods approaches (4th ed.). SAGE Publications Inc.

Dávila, C. (2001). Teorías Organizacionales y Administración (2da ed.). McGraw-Hill 
Drucker, P. F. (1985). Innovation and entrepreneurship. Practice and principles. Harper \& Row Publisher.

Evan, W. M. (1967). La Órbita de la Organización: Hacia una Teoría de las Relaciones Interorganizacionales. OMEBA.

Fayol, H. (1975). Administración Industrial y General (20 ed). Herrero Hermanos.

Field, A. (2009). Discovering Statistics (3 ${ }^{\mathrm{a}}$ Ed.). Sage Publications Ltd

Fierro-Moreno, E., \& Mercado-Salgado, P. (2012). La Innovación Organizativa y sus Predictores Desde la Teoría de Recursos y Capacidades. Administración y $\begin{array}{lll}\text { Organizaciones. } & \text { 93-115. }\end{array}$ https://rayo.xoc.uam.mx/index.php/Rayo/article/view/139

Global Entrepreneurship Monitor (GEM). Global Report 2017/18. Global Entrepreneurship Research Association (GERA) (2018)

Hair, J. F., Black, W.C., Barbin, B., \& Anderson, R. (2014). Multivariate Data Analysis (7 ed.), 601-622; 197-199. Pearson.

Hernández-Sampieri, R., Fernández-Collado, C., \& Baptista-Lucio, P. (2014). Metodología de la Investigación (4a ed). McGraw Hill.

Ho, R. (2006). Handbook of Univariate and Multivariate Data Analysis with IBM SPSS (1 ed.). Chapman and Hall/CRC

Hofstede, G., y Hofstede, G.J. (2005) Cultures and Organizations: Software of the Mind, Revised and Expanded (2 ${ }^{\mathrm{a}}$ Ed.), McGraw-Hill.

Madrigal, B. E., Núñez, M. A., Madrigal, R. y Arechavala, R. (2019). Innovative Ability Mission of the Enterprising University. Journal of Humanities, Arts and Social Science, 3(2), 32-42. DOI:10.26855/jhass.2019.09.001

Madrigal, B.E. y Núñez, M.A. (2020). Emprendimiento e Innovación en la 4ta revolución industrial. McGraw-Hill.

Martínez, M. y Fierro, E. (2018). Aplicación de la Técnica PLS-SEM en la Gestión del Conocimiento: un Enfoque Técnico Práctico. RIDE Revista Iberoamericana para la 
Investigación y el Desarrollo Educativo, 8(16), 130-164. https://doi.org/10.23913/ride.v8i16.336

McClelland, D. (1961). The Achievement Society. Van Nostrand.

Muñoz-Osuna, F. O., Medina-Rivilla, A., \& Guillén-Lúgigo, M. (2016). Jerarquización de Competencias Genéricas Basadas en las Percepciones de Docentes Universitarios. Educación Química, 27(2), 126-132 https://doi.org/10.1016/j.eq.2015.11.002

Ochoa-Díaz, H., Ríos-Millán, A. M., \& Solano-Castillo, N. (2019). La Innovación como Competencia Central en la Internacionalización de las Firmas Latinoamericanas: El Proceso de Bico Internacional, Empresa del Grupo Carvajal S.A. Estudios Gerenciales, 27(119), 13-32. https://doi.org/10.1016/S0123-5923(11)70155-3

Olivares-Olivares, S. L., Adame-Torres, E., Avila-Palet, J. E., Turrubiates-Corolla, M. L., López-Cabrera, M. V., \& Valdez-García, J. E. (2019). Valor Percibido de una Experiencia de Inmersión Educativa para el Desarrollo de Competencias Transversales: Semana i. Educación Médica, 20(2), 93-99. https://doi.org/10.1016/j.edumed.2018.04.015

Provan, K. G., \& Milward, H. B. (1995). A Preliminary Theory of Interorganizational Network Effectiveness: A Comparative Study of Four Community Mental Health Systems. Administrative Science Quarterly, 40(1),1-33. https://doi.org/10.2307/2393698

Rangel-Torrijo, H. (2015). Metodologías para la Innovación Curricular Universitaria Basada en el Desarrollo de Competencias Oscar Corvalán, Jacques Tardif y Patricio Moreno (Coordinadores) (2013) México, ANUIES. Perfiles Educativos, 37(147), 228-234. https://doi.org/10.1016/j.pe.2015.11.003

Ringle, C.M., Wende, S. y Becker, J.M. (2015). SmartPLS 3. SmartPLS GmbH, Boenningstedt, 
Rochina, M., \& Rodriguez, J. (2019). Innovation Drivers in Ecuadorian Manufacturing. Managemenet Research, 17(4), 510-539. https://doi.org/10.1108/MRJIAM-11-2018$\underline{0886}$

Schumpeter, J. (1994). The Theory of Economic Development. Oxford University Press

Schumpeter, J. A. (1934). The Theory of Economic Development. Harvard University Press.

Taylor, F. (1975). Principios de la Administración Científica (20a ed.). Herrero Hermanos.

Watts, F., García-Carbonell, A., \& Andreu-Andrés, M. Á. (eds.). (2013). Innovation Competencies Development, INCODE Barometer and User Guide. Turku University of Applied Sciences

Weber, M. (1992). Economía y Sociedad. Fondo de Cultura Económica. Cd. de Méxoco.

\section{Cómo citar:}

Gómez Álvarez, P. ., Núñez Ramírez, M. ., \& Esparza García, I. . (2021). Modelo estructural de las competencias de innovación en Latinoamérica. Evidencia en México, Colombia y Bolivia. Revista De Investigación Académica Sin Frontera: División De Ciencias Económicas Y Sociales, (36).

https://doi.org/10.46589/rdiasf.vi36.426

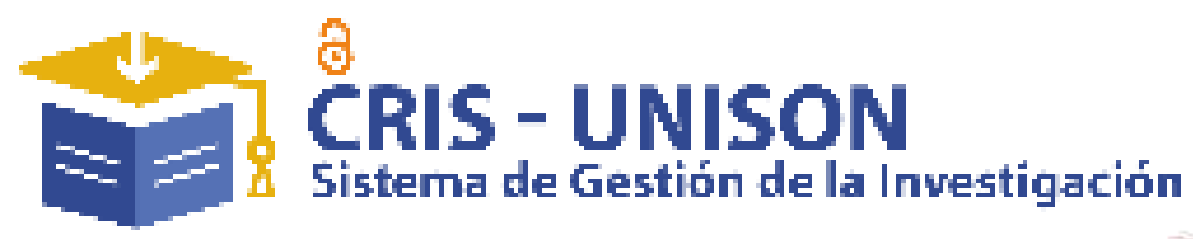

D Dienet

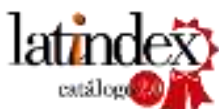

inciciofactor

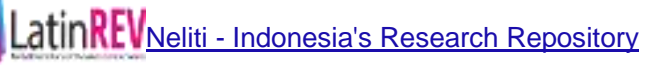

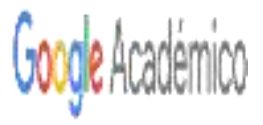

REDIB

IJIFACTOR

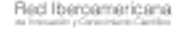

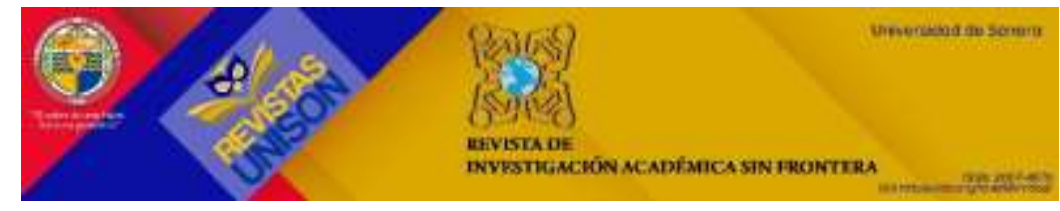


Año 14 / Núm. 36 / julio-diciembre 2021 